\title{
Characterization of adrenal metastatic cancer using FDG PET/CT
}

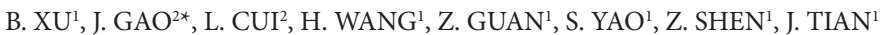 \\ ${ }^{1}$ Department of Nuclear Medicine, Chinese PLA General Hospital, Beijing, People's Republic of China; ${ }^{2}$ Department of Urology, Chinese PLA \\ General Hospital, Beijing, People's Republic of China \\ ${ }^{\star}$ Correspondence: jiangpgao@163.com
}

Received August 8, 2011 / Accepted September 5, 2011

\begin{abstract}
The adrenal gland is a common location for metastasis from a primary tumor in another organ. This study evaluated the properties of adrenal lesions in cancer and non-cancer patients and investigated what variables may help predict adrenal metastasis. This retrospective study used ${ }^{18}$ fluorodeoxyglucose PET/CT on 371 patients with adrenal lesions (N $=260$ with a primary tumor and $\mathrm{N}=111$ with an unknown primary tumor). Parameters such as the presence of a tumor, nodule, enlarged adrenal, maximum standardized uptake ( $\mathrm{SUV}_{\max }$ ratio) were evaluated. Univariate and multivariate analysis were used to identify variables that may predict risk of adrenal metastasis. Subjects with adrenal metastasis versus those without had a higher frequency of primary lung tumors ( $53.7 \%$ versus $28.6 \%$, respectively; $\mathrm{P} \leq 0.001)$ but a lower frequency of gastrointestinal cancer ( $9.3 \%$ versus $20.4 \%$, respectively; $\mathrm{P}=0.014$ ). The frequency of other abnormalities including nodules and enlarged adrenals were similar between cancer and non-cancer subjects. A higher proportion of subjects with adrenal metastasis regardless whether the primary tumor site in the lung, gastrointestinal track, or liver had $\mathrm{SUV}_{\max }$ ratio $>2.5$ versus those with no adrenal metastasis. In this cohort of subjects, the greatest proportion of subjects with adrenal metastasis was those with primary lung cancer. Univariate and multivariate analysis indicated that age, $\mathrm{SUV}_{\max }$ ratio, and the presence of metastasis in multiple organs were independent variables for having adrenal metastasis. In this study, FDG PET/CT was useful in characterizing adrenal lesions including determining whether they were benign or malignant. This technology allowed us to identify characteristics that may useful in predicting adrenal metastasis and cancer severity.
\end{abstract}

Key words: fluorodeoxyglucose, positron emission tomography, computed tomography, adrenal metastasis, cancer

The adrenal gland is a common site for metastasis in patients with cancer with the rate of metastasis being between $25 \%$ to $75 \%$ depending on the type and size of the primary tumor [1]. The most common malignant lesions that metastasize to the adrenal gland include malignant melanoma, breast, lung, kidney, esophagus, pancreases, liver, stomach, and colon cancers $[1,2]$. However diagnosis of an adrenal lesion as malignant or benign can be problematic. For example, patients with adrenal metastasis are usually asymptomatic and even in patients with a history of cancer, $50 \%$ of adrenal tumors are not malignant [3]. Moreover, although most adrenal masses in patients are benign, about $2.5 \%$ are malignant [1]. Accurate characterization of these adrenal lesions in cancer patients is critical for accurate diagnosis, therapeutic strategy, and disease prognosis [2].

There are multiple methods for discerning benign from malignant lesions. The percutaneous biopsy is an effective procedure, however it is invasive and technically difficult [4]. Monitoring cortisol secretion is useful although not all malignant tumors result in increased hormone secretion and the increased secretion may result from other non-cancerous causes [2]. Imaging of the adrenal gland to diagnose a mass is typically accomplished using computed tomography (CT) or magnetic resonance imaging (MRI). CT measures attenuation to differentiate between benign and malignant lesions, but the use of attenuation is not always possible depending upon the characteristic of the lesions [5,6]. The use of MRI can be problematic since the malignant and benign lesion signal intensity overlap [7].

${ }^{18} \mathrm{~F}$-Fluorodeoxglucose positron emission tomography (PET) with CT (FDG PET/CT) is also used to investigate potential adrenal cancer and provides anatomo-metabolic information and has certain advantages over other techniques. It has faster attenuation and lower mismatches compared 
with PET alone, and malignancies may be detected earlier since metabolic changes in the tissue may precede anatomical changes [8]. Several studies have shown that FDG PET/CT high sensitivity, specificity and accuracy (all $>90 \%$ ) for detecting adrenal metastatic disease [8-10].

FDG PET/CT attributes make it not only useful for determining if a mass is benign or cancerous but it also can be used as a non-invasive method to help characterize the cancer itself which may facilitate the physician in diagnosis and determination of treatment strategies. However, few studies have investigated this issue. The objective of this study was to use FDG PET/CT to characterize the properties of adrenal lesions in patients with cancer. Specifically, we investigated whether there are differences between malignancies and benign lesions, are tumor characteristics associated with the location of the primary tumor, and are there predictors for adrenal metastasis. We also further evaluated the effectiveness of FDG PET/CT in distinguishing between benign and malignant lesions.

\section{Patients and methods}

This is a retrospective study of FDG PET/CT scan performed from 2007 to 2010. Patients who visited the Chinese PLA General hospital either with or without a history of cancer prior to the FDG PET/CT test were recruited into the study. This study was approved by the Institutional Review Board of Chinese PLA General Hospital, and the requirement of informed consent was waived due to the retrospective nature of this study.

Study population. Eligible patients had an abnormal adrenal lesion as determined by an initial PET/CT scan which was diagnosed as adrenal metastasis (for those with known primary tumors), adrenal adenoma, or adrenal hyperplasia by a pathological follow-up (6 to 30 months following the initial PET/CT). Patients were excluded from the study if they had been treated for malignant or benign lesions in the adrenal gland, had diabetes, or any other disorder that may affect glucose metabolism.

Image collection and analysis. All patients fasted for $\geq 4$ hours prior to FDG PET/CT. Images were acquired 60 minutes following the administration of $55.5 \mathrm{MBq} / \mathrm{kg}{ }^{18} \mathrm{~F}-\mathrm{FDG}$ using the Siemens Biograph 64 HR (Siemens Medical Solutions USA, Molecular Imaging, Hoffman Estates, IL, USA). The CT scanning parameters were as follows: a low-dose CT (LDCT) scan was performed at $120 \mathrm{kV}, 100 \mathrm{mAs}, 0.8 \mathrm{~s}$ rotation, with a $1.25 \mathrm{~mm}$ slice width, and pitch of 0.9. The PET data were acquired immediately after the CT scan in 3-D mode for 2.5 $\mathrm{min} /$ bed and 3 or 7 different bed positions. The scan covered from the bottom of the chin to the bottom of the pelvis. The PET images, including axial, sagittal and coronal images, were reconstructed by Fourier rebinning (FORE) ordered subset expectation maximization (OSEM) algorithm with attenuation correction.

Image interpretation. The region of interest (ROI) was identified and was large enough to cover more than half the adrenal mass but did not include any peripheral areas so as to avoid partial volume effects. A similar sized ROI was chosen on a non-affected liver region. The maximum standardized uptake values $\left(\mathrm{SUV}_{\max }\right)$ were calculated for both ROIs and the ratio of the lesion to the normal tissue was calculated.

Statistical analysis. Age was expressed by mean with standard deviation (SD) and compared between groups by the independent two samples t-test. Other categorical variables were expressed by count with percentage, and the associations between categorical variables were tested with the Fisher's exact test. The impact factors of adrenal metastasis were expressed by their odds ratios with the $95 \%$ confidence interval (CI) in the univariate and multivariate logistic regression models. The cutoff point of SUV ratio (1.25) in the predictions for adrenal metastasis was determined by the Youden's index (the maximum of sensitivity+specificity-1) in the ROC analysis. The variables with $P$-value less than 0.1 were included into the multivariate logistic regression models according to the selections by forward conditional method. All statistical analyses were set with a significance level of 0.05 and performed using SPSS 15.0 statistics software (SPSS Inc, Chicago, IL, USA).

\section{Results}

FDG PET/CT examination was performed on 11570 subjects, including those with primary cancer $(\mathrm{N}=3882)$ and those without cancer $(\mathrm{N}=7688)$. Of all the patients examined, 374 subjects had adrenal gland lesions, and 3 young subjects ( 2 to 3 years of age) were excluded. The characteristics of the adrenal lesions of remaining 371 subjects were analyzed $(\mathrm{N}$ $=260$ with a non-adrenal primary tumor and $\mathrm{N}=111$ with unknown primary cancer).

Demographics and adrenal lesion characteristics. The 2 groups of patients were similar with regard to age and gender (Table 1), and the majority of subjects in both groups were male. Over half $(62.3 \%)$ the patients with a non-adrenal primary tumor had a malignant adrenal lesion (Table 1). The types of non-adrenal primary tumors included lung (44.2\%), gastrointestinal (13.5\%), and liver cancers $(8.8 \%)$. On the contrary, patients with unknown primary tumors did not have malignant adrenal lesions; their lesions were either benign (55.5\%) or adrenal hyperplasia (45.0\%) (Table 1).

Significant differences were observed in FDG PET/CT characteristics between subjects with or without a known primary tumor. The proportion of patients with a tumor mass (in either the right or left adrenal) was small in both groups (< $8 \%$ ) but was significantly higher for the subjects with a known primary tumor compared to those without $(P$-value $\leq 0.024)$ (Table 1). A similar proportion of patients in the 2 groups had lesions that were characterized as nodules, enlarged adrenal glands, or normal (Table 1). More patients had normal right than left adrenals.

The SUV $V_{\max }$ ratio was significantly higher in subjects with a known primary tumor than those without (Table 1). For 


\begin{tabular}{|c|c|c|c|c|}
\hline & & \multicolumn{2}{|c|}{ With known primary tumor } & \multirow[b]{2}{*}{$P$-value } \\
\hline & & $\begin{array}{c}\text { Yes } \\
(N=260)\end{array}$ & $\begin{array}{c}\text { No } \\
(\mathrm{N}=111)\end{array}$ & \\
\hline \multicolumn{5}{|l|}{ Demographics } \\
\hline Age (year) & & $61.7 \pm 13.3$ & $63.3 \pm 12.4$ & 0.280 \\
\hline \multirow[t]{3}{*}{$\begin{array}{l}\text { Male } \\
\text { Abnormalities in adrenals }\end{array}$} & & $181(69.6 \%)$ & $80(72.1 \%)$ & 0.710 \\
\hline & Adrenal metastasis or cancer & $162(62.3 \%)$ & $0(0.0 \%)$ & $<0.001^{*}$ \\
\hline & Benign adrenal lesion & $43(16.5 \%)$ & $61(55.0 \%)$ & \\
\hline Adrenal hyperplasia & & $55(21.2 \%)$ & $50(45.0 \%)$ & \\
\hline \multirow{4}{*}{$\begin{array}{l}\text { PET/CT characteristics } \\
\text { Left adrenal }\end{array}$} & Tumor mass & $16(6.2 \%)$ & $1(0.9 \%)$ & $0.026^{*}$ \\
\hline & Nodule & $96(36.9 \%)$ & $50(45.0 \%)$ & \\
\hline & Enlarged & $80(30.8 \%)$ & $40(36.0 \%)$ & \\
\hline & Normal & $68(26.2 \%)$ & $20(18.0 \%)$ & \\
\hline \multirow{4}{*}{ Right adrenal } & Tumor mass & $20(7.7 \%)$ & $1(0.9 \%)$ & $0.024^{*}$ \\
\hline & Nodule & $51(19.6 \%)$ & $17(15.3 \%)$ & \\
\hline & Enlarged & $32(12.3 \%)$ & $16(14.4 \%)$ & \\
\hline & Normal & $157(60.4 \%)$ & $77(69.4 \%)$ & \\
\hline \multirow[t]{4}{*}{$\mathrm{SUV}_{\max }$ ratio $^{*}$} & $>2.5$ & $60(26.3 \%)$ & $2(1.9 \%)$ & $<0.001^{*}$ \\
\hline & $\leq 2.5$ & $103(45.2 \%)$ & $27(25.2 \%)$ & \\
\hline & absence of accumulated radioactivity & $65(28.5 \%)$ & $78(72.9 \%)$ & \\
\hline & Unknown & - & $111(100 \%)$ & \\
\hline \multirow[t]{4}{*}{ Primary tumor } & Lung cancer & $115(44.2 \%)$ & - & \\
\hline & Gastrointestinal cancer & 35 (13.5\%) & - & \\
\hline & Liver cancer & $23(8.8 \%)$ & - & \\
\hline & Other & 87 (33.5\%) & - & \\
\hline
\end{tabular}

"indicates a significant association between the corresponding variable and group was observed. *There were 36/371 (9.70) missing values in SUV.

example, $26.3 \%$ and $1.9 \%$ of subjects with or without a known primary tumor, respectively, had a $\mathrm{SUV}_{\text {max }}$ ratio of $>2.5(P<$ $0.001)$; more patients with unknown a primary tumor $(72.9 \%$ versus $28.5 \%$ ) had evidence of radioactive fluorodeoxglucose accumulation (Table 1).

Associations between demographics and PET/CT characteristics in subjects with a primary tumor. Patients with adrenal metastasis were younger than those whose primary tumor had not metastasized to the adrenal gland. (60.1 versus 64.4 years, respectively; $P=0.01$ ) (Table 2 ). For both adrenals, the proportion of patients with nodule or tumor mass was higher in those with a adrenal metastasis than in those without (Table 2). The proportion of patients with a $\mathrm{SUV}_{\max }$ ratio of $>$ 2.5 (the accepted value for distinguishing between benign and cancerous lesions) was greater for patients with adrenal metastasis $(44.4 \%)$ compared to those without adrenal metastasis $(1.1 \% ; P<0.001)$ (Table 2). A great proportion of patients with adrenal metastasis had metastasized tumors in other parts of their body (75.3\% for patients with adrenal metastasis and $36.7 \%$ with no adrenal metastasis; $P<0.001$ ).

About half the patients with adrenal metastasis (53.7\%) had primary lung cancer, and less than $10 \%$ of patients had the primary tumors in the gastrointestinal track or the liver (Table 2). Subjects with adrenal metastasis versus those without had a higher frequency of primary lung tumors (53.7\% versus $28.6 \%$, respectively; $\mathrm{P} \leq 0.001$ ) but a lower frequency of gastrointestinal cancer (9.3\% versus $20.4 \%$, respectively; $\mathrm{P}=0.014)$. 
For patients with primary tumors in the lung, gastrointestinal track, or the liver, the frequency of either the left or right adrenal having a nodule or tumor mass was greater in those subjects with adrenal metastasis (Table 2), and this reached statistical significance for patients with lung cancer. A higher proportion of subjects with lung and gastrointestinal primary tumors and adrenal metastasis had a SUV ${ }_{\max }$ ratio $>2.5$ than the same group of patients without adrenal metastasis $(P \leq$ 0.001 ). For the 3 subgroups of subjects, a higher proportion of patients with adrenal metastasis also had metastasis in other organs compared to those subjects without adrenal metastasis. This was statistically significant for subjects with lung and gastrointestinal primary tumors $(P \leq 0.009)$. Patients with adrenal metastasis and gastrointestinal cancer were significantly younger than those without adrenal metastasis (mean age 58.2 versus 68.4 years, $P=0.017$ ).

Summary of univariate analysis of predictors of adrenal metastasis. The odds ratios from the univariate logistic regression models analysis indicated that the six variables of age, left and right adrenal FDG PET/CT characteristics, $\mathrm{SUV}_{\max }$ ratio, metastasis of the primary tumor to multiple locations, and location of the primary tumor were significant predictors of adrenal metastasis $(P \leq 0.011)$ (Table 3$)$. However, only age, $\mathrm{SUV}_{\max }$ ratio, and the presence metastasis in multiple organs were independent variables for adrenal metastasis according to the selections by forward conditional method (Table 4). There was one exception that age was excluded from the multivariate analysis of lung cancer in Table 4 since it had no independent impact on adrenal metastasis. For all subjects after controlling for the $\mathrm{SUV}_{\max }$ ratio and multiple metastatic locations, the risk for developing adrenal metastasis decreased slightly by each year of increasing age $(\mathrm{OR}=0.97, P<0.021)$. Controlling for age and $\mathrm{SUV}_{\max }$ ratio, subjects with metastasis in multiple locations had a significantly higher risk of adrenal metastasis than those with no additional metastasis $(\mathrm{OR}=$ $4.19, P<0.001)$. After controlling for the other 2 variable, subjects with a SUV ${ }_{\text {max }}$ ratio between $>0$ and $\leq 2.5$ or $>2.5$ had significantly higher risk of adrenal metastasis than those who had no observable SUV $(\mathrm{OR}=13.71, P<0.001$ and $\mathrm{OR}$ $=461.54, P<0.001$, respectively).

$\mathrm{SUV}_{\max }$ ratio and multiple metastasis locations were also independent variables for risk for adrenal metastasis in the cohort of patients with lung cancer (Table 3). After controlling for $\mathrm{SUV}_{\max }$ ratio, the subjects with multiple metastatic sites versus those with no additional cancer sites had higher risk of adrenal metastasis $(\mathrm{OR}=4.12, P=0.041)$. Similarly, patients with $\mathrm{SUV}_{\max }$ ratio $>0$ and $\leq 2.5$ as well as those with values $>$ 2.5 had a higher risk of adrenal metastasis than subjects with no measurable SUV (OR $=33.85, P<0.001 \mathrm{OR}=300.96, P<$ 0.001 , respectively).

The variables of age, $\mathrm{SUV}_{\max }$ ratio, and multiple metastatic sites were significant predictors of adrenal metastasis in patients with gastrointestinal cancer. Only age and $\mathrm{SUV}_{\max }$ ratio were included in the multivariate analysis. After controlling for age, the $\mathrm{SUV}_{\max }$ ratio was found to be an independent

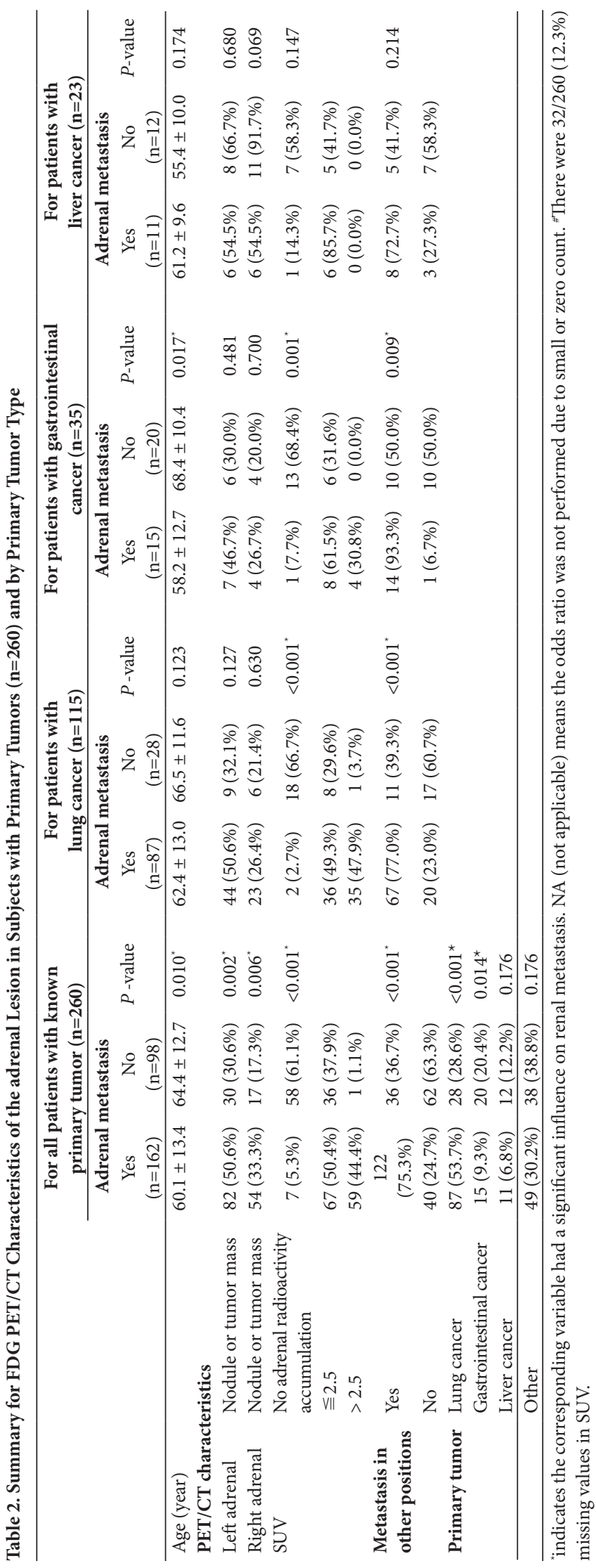


Table 3. Summary univariate logistic regression analysis.

\begin{tabular}{|c|c|c|c|c|c|c|c|c|c|}
\hline & & \multicolumn{2}{|c|}{$\begin{array}{l}\text { All Cancer Subjects } \\
\qquad(\mathrm{N}=\mathbf{2 6 0}) \\
\end{array}$} & \multicolumn{2}{|c|}{$\begin{array}{c}\text { Subjects with } \\
\text { lung cancer }(\mathrm{N}=115)\end{array}$} & \multicolumn{2}{|c|}{$\begin{array}{c}\text { Subjects with } \\
\text { gastrointestinal cancer } \\
(\mathrm{N}=35)\end{array}$} & \multicolumn{2}{|c|}{$\begin{array}{c}\text { Subjects with } \\
\text { liver cancer }(N=23)\end{array}$} \\
\hline & & OR $(95 \% \mathrm{CI})$ & $P$-value & OR $(95 \% \mathrm{CI})$ & $P$-value & OR $(95 \% \mathrm{CI})$ & $P$-value & OR (95\% CI) & $P$-value \\
\hline \multicolumn{10}{|l|}{ Demographics } \\
\hline Age (year) & & $0.98(0.96,0.99)$ & $0.011^{*}$ & $0.97(0.94,1.01)$ & 0.142 & $0.92(0.86,0.99)$ & $0.026^{*}$ & $1.07(0.97,1.17)$ & 0.177 \\
\hline Gender & Female & - & & - & & - & & - & \\
\hline Male & & $0.58(0.33,1.03)$ & 0.061 & $1.06(0.43,2.57)$ & 0.905 & $0.31(0.05,1.95)$ & 0.210 & $\begin{array}{c}0.91(0.05 \\
16.54)\end{array}$ & 0.949 \\
\hline \multicolumn{10}{|c|}{ PET/CT characteristic } \\
\hline \multirow[t]{2}{*}{ Left adrenal } & Normal or enlarged & - & & - & & - & & - & \\
\hline & nodule or tumor mass & $2.32(1.37,3.94)$ & $0.002^{*}$ & $2.16(0.88,5.30)$ & 0.093 & $2.04(0.51,8.23)$ & 0.316 & $1.67(0.31,9.01)$ & 0.553 \\
\hline \multirow[t]{2}{*}{ Right adrenal } & Normal or enlarged & - & & - & & - & & - & \\
\hline & nodule or tumor mass & $2.38(1.29,4.41)$ & $0.006^{*}$ & $1.32(0.47,3.66)$ & 0.596 & $1.45(0.30,7.09)$ & 0.643 & $9.17(0.86,97.69)$ & 0.066 \\
\hline \multirow[t]{3}{*}{$\mathrm{SUV}_{\max }$ ratio } & $>2.5$ & $\begin{array}{c}488.86(58.30 \\
4,098.89)\end{array}$ & $<0.001^{*}$ & $\begin{array}{c}315.00(26.73 \\
3,712.37)\end{array}$ & $<0.001^{*}$ & NA & & NA & \\
\hline & $\leqq 2.5$ & $15.42(6.38,37.28)$ & $<0.001^{*}$ & $40.50(7.78,210.79)$ & $<0.001^{*}$ & $17.33(1.75,171.66)$ & $0.015^{*}$ & $8.40(0.76,93.34)$ & 0.083 \\
\hline & $\begin{array}{l}\text { absence of radioactiv- } \\
\text { ity accumulation }\end{array}$ & - & & - & & - & & - & \\
\hline \multirow{2}{*}{$\begin{array}{l}\text { Metastasis in } \\
\text { other positions }\end{array}$} & Yes & $5.25(3.05,9.05)$ & $<0.001^{*}$ & $5.18(2.09,12.84)$ & $<0.001^{*}$ & $14.00(1.54,127.62)$ & $0.019^{*}$ & $3.73(0.65,21.58)$ & 0.141 \\
\hline & No & - & & - & & - & & - & \\
\hline \multirow{4}{*}{$\begin{array}{l}\text { Primary } \\
\text { tumor }\end{array}$} & Lung cancer & $2.41(1.32,4.39)$ & $0.004^{*}$ & & & & & & \\
\hline & Gastrointestinal cancer & $0.58(0.26,1.28)$ & 0.180 & & & & & & \\
\hline & Liver cancer & $0.71(0.28,1.79)$ & 0.468 & & & & & & \\
\hline & Other & - & & & & & & & \\
\hline
\end{tabular}

- Reference group. indicates the corresponding variable had a significant influence on adrenal metastasis. NA (not applicable) means the odds ratio was not performed due to small or zero count.

Table 4. Summary for the impact factors of adrenal metastasis by multivariate logistic regression models.

\begin{tabular}{|c|c|c|c|c|c|c|c|}
\hline & & \multicolumn{2}{|c|}{$\begin{array}{l}\text { For all patients } \\
\text { with primary tumor }(\mathrm{n}=260)^{\mathrm{a}}\end{array}$} & \multicolumn{2}{|c|}{$\begin{array}{l}\text { For patients with } \\
\text { lung cancer }(n=115)^{b}\end{array}$} & \multicolumn{2}{|c|}{$\begin{array}{c}\text { For patients with } \\
\text { gastrointestinal cancer } \\
\qquad(\mathbf{n}=35)^{\mathrm{c}}\end{array}$} \\
\hline & & OR $(95 \% \mathrm{CI})$ & P-value & OR $(95 \% \mathrm{CI})$ & $\mathrm{P}$-value & OR $(95 \% \mathrm{CI})$ & $\mathrm{P}$-value \\
\hline Age (year) & & $0.97(0.94,0.99)$ & $0.021^{*}$ & & & $0.91(0.83,1.00)$ & 0.060 \\
\hline \multirow[t]{3}{*}{ SUV $_{\max }$ ratio } & $>2.5$ & $461.54(52.72,4,040.50)$ & $<0.001^{*}$ & $\begin{array}{c}300.96(24.13 \\
3,754.25)\end{array}$ & $<0.001^{*}$ & NA & \\
\hline & $<2.5$ & $13.71(5.38,34.92)$ & $<0.001^{*}$ & $33.85(6.20,184.68)$ & $<0.001^{*}$ & $20.92(1.62,270.40)$ & $0.020^{*}$ \\
\hline & $\begin{array}{l}\text { absence of radioactivity } \\
\text { accumulation }\end{array}$ & - & & - & & - & \\
\hline \multirow[t]{2}{*}{$\begin{array}{l}\text { Metastasis in other } \\
\text { positions }\end{array}$} & Yes & $4.19(1.93,9.13)$ & $<0.001^{*}$ & $4.12(1.06,15.98)$ & $0.041^{*}$ & & \\
\hline & No & - & & - & & & \\
\hline
\end{tabular}

Reference group. "indicates the corresponding variable had a significant influence on adrenal metastasis. NA (not applicable) means the odds ratio was not performed due to small or zero count. ${ }^{\mathrm{a} P}$-value $=\mathbf{0 . 3 2 1}$ in Hosmer and Lemeshow test indicates the multivariate model fit well. ${ }^{\mathrm{b}} \mathrm{P}$-value $=\mathbf{0 . 6 8 6}$ in Hosmer and Lemeshow test indicates the multivariate model fit well. ${ }^{c} \mathbf{P}$-value $=0.136$ in Hosmer and Lemeshow test indicates the multivariate model fit well

predictor of the risk of adrenal metastasis $(\mathrm{OR}=20.93, P=$ 0.02). For subjects with liver cancer, no measured variable was significant in the univariate analysis, and the sample size was too small $(\mathrm{N}=23)$ to perform multivariate analysis, therefore they were not included in Table 4.
Summary of multivariate analysis of predictors of adrenal metastasis. For patients with non-adrenal primary cancer, multivariate analysis indicated that the $\mathrm{SUV}_{\max }$ ratio, multiple metastatic sites, and age were independent impact factors of adrenal metastasis. Various combinations of the three 
Table 5. The sensitivity, specificity, PPV, and NPV of the predictions for adrenal metastasis by age, $\mathrm{SUV}_{\max }$ ratio, and metastasis in other positions.

\begin{tabular}{|c|c|c|c|c|c|c|c|c|}
\hline & & \multicolumn{2}{|c|}{ Adrenal metastasis } & \multirow{2}{*}{ Sensitivity } & \multirow{2}{*}{ Specificity } & \multirow{2}{*}{ PPV } & \multirow{2}{*}{ NPV } & \multirow{2}{*}{ Accuracy } \\
\hline & & Yes & No & & & & & \\
\hline \multicolumn{9}{|l|}{ For all patients with primary tumor } \\
\hline \multirow[t]{2}{*}{ 1. $\mathrm{SUV}_{\max }$ ratio $>0$} & Yes & 126 & 37 & $77.8 \%$ & $62.2 \%$ & $77.3 \%$ & $62.9 \%$ & $71.9 \%$ \\
\hline & No & 36 & 61 & & & & & \\
\hline \multirow[t]{2}{*}{ 2. $\mathrm{SUV}_{\max }$ ratio $>1.25$} & Yes & 113 & 12 & $69.8 \%$ & $87.8 \%$ & $90.4 \%$ & $63.7 \%$ & $76.5 \%$ \\
\hline & No & 49 & 86 & & & & & \\
\hline \multirow{2}{*}{$\begin{array}{l}\text { 3. } \mathrm{SUV}_{\max } \text { ratio }>1.25 \text { or metastasis in other } \\
\text { positions }\end{array}$} & Yes & 148 & 40 & $91.4 \%$ & $59.2 \%$ & $78.7 \%$ & $80.6 \%$ & $79.2 \%$ \\
\hline & No & 14 & 58 & & & & & \\
\hline \multirow{2}{*}{$\begin{array}{l}\text { 4. } \mathrm{SUV}_{\text {max }} \text { ratio }>1.25 \text { or metastasis in other } \\
\text { positions or age }<55 \text { years }\end{array}$} & Yes & 155 & 56 & $95.7 \%$ & $42.9 \%$ & $73.5 \%$ & $85.7 \%$ & $75.8 \%$ \\
\hline & No & 7 & 42 & & & & & \\
\hline \multirow{2}{*}{$\begin{array}{l}\text { 5. } \mathrm{SUV}_{\text {max }} \text { ratio }>1.25 \text { or metastasis in other } \\
\text { positions or age }<60 \text { years }\end{array}$} & Yes & 156 & 64 & $96.3 \%$ & $34.7 \%$ & $70.9 \%$ & $85.0 \%$ & $73.1 \%$ \\
\hline & No & 6 & 34 & & & & & \\
\hline \multirow{2}{*}{$\begin{array}{l}\text { 6. } \mathrm{SUV}_{\text {max }} \text { ratio }>0 \text { or metastasis in other } \\
\text { positions }\end{array}$} & Yes & 153 & 55 & $94.4 \%$ & $43.9 \%$ & $73.6 \%$ & $82.7 \%$ & $75.4 \%$ \\
\hline & No & 9 & 43 & & & & & \\
\hline \multirow{2}{*}{$\begin{array}{l}\text { 7. } \mathrm{SUV}_{\text {max }} \text { ratio }>0 \text { or metastasis in other } \\
\text { positions or age }<55\end{array}$} & Yes & 159 & 67 & $98.1 \%$ & $31.6 \%$ & $70.4 \%$ & $91.2 \%$ & $73.1 \%$ \\
\hline & No & 3 & 31 & & & & & \\
\hline \multirow{2}{*}{$\begin{array}{l}\text { 8. } \mathrm{SUV}_{\text {max }} \text { ratio }>0 \text { or metastasis in other } \\
\text { positions or age }<60\end{array}$} & Yes & 160 & 74 & $98.8 \%$ & $24.5 \%$ & $68.4 \%$ & $92.3 \%$ & $70.8 \%$ \\
\hline & No & 2 & 24 & & & & & \\
\hline \multicolumn{9}{|l|}{ For the patients with lung cancer } \\
\hline \multirow{2}{*}{ 9. $\mathrm{SUV}_{\max }$ ratio $>0$} & Yes & 71 & 9 & $81.6 \%$ & $67.9 \%$ & $88.8 \%$ & $54.3 \%$ & $78.3 \%$ \\
\hline & No & 16 & 19 & & & & & \\
\hline \multirow[t]{2}{*}{ 10. $\mathrm{SUV}_{\max }$ ratio $>1.25$} & Yes & 62 & 4 & $71.3 \%$ & $85.7 \%$ & $93.9 \%$ & $49.0 \%$ & $74.8 \%$ \\
\hline & No & 25 & 24 & & & & & \\
\hline \multirow{2}{*}{$\begin{array}{l}\text { 11. } \mathrm{SUV}_{\text {max }} \text { ratio }>0 \text { or metastasis in other } \\
\text { positions }\end{array}$} & Yes & 84 & 15 & $96.6 \%$ & $46.4 \%$ & $84.8 \%$ & $81.3 \%$ & $84.3 \%$ \\
\hline & No & 3 & 13 & & & & & \\
\hline \multirow{2}{*}{$\begin{array}{l}\text { 12. } \mathrm{SUV}_{\text {max }} \text { ratio }>1.25 \text { or metastasis in other } \\
\text { positions }\end{array}$} & Yes & 81 & 12 & $93.1 \%$ & $57.1 \%$ & $87.1 \%$ & $72.7 \%$ & $84.3 \%$ \\
\hline & No & 6 & 16 & & & & & \\
\hline \multicolumn{9}{|l|}{ For the patients with gastrointestinal cancer } \\
\hline \multirow[t]{2}{*}{ 13. $\mathrm{SUV}_{\max }$ ratio $>0$} & Yes & 12 & 6 & $80.0 \%$ & $70.0 \%$ & $66.7 \%$ & $82.4 \%$ & $74.3 \%$ \\
\hline & No & 3 & 14 & & & & & \\
\hline \multirow[t]{2}{*}{ 14. $\mathrm{SUV}_{\max }$ ratio $>1.25$} & Yes & 12 & 2 & $80.0 \%$ & $90.0 \%$ & $85.7 \%$ & $85.7 \%$ & $85.7 \%$ \\
\hline & No & 3 & 18 & & & & & \\
\hline \multirow{2}{*}{ 15. $\mathrm{SUV}_{\max }$ ratio $>0$ or age $<55$} & Yes & 13 & 7 & $86.7 \%$ & $65.0 \%$ & $65.0 \%$ & $86.7 \%$ & $74.3 \%$ \\
\hline & No & 2 & 13 & & & & & \\
\hline 16. $\mathrm{SUV}_{\max }$ ratio $>0$ or age $<60$ & Yes & 13 & 9 & $86.7 \%$ & $55.0 \%$ & $59.1 \%$ & $84.6 \%$ & $68.6 \%$ \\
\hline & No & 2 & 11 & & & & & \\
\hline 17. $\mathrm{SUV}_{\max }$ ratio $>1.25$ or age $<55$ & Yes & 13 & 3 & $86.7 \%$ & $85.0 \%$ & $81.3 \%$ & $89.5 \%$ & $85.7 \%$ \\
\hline & No & 2 & 17 & & & & & \\
\hline 18. $\mathrm{SUV}_{\max }$ ratio $>1.25$ or age $<60$ & Yes & 13 & 5 & $86.7 \%$ & $75.0 \%$ & $72.2 \%$ & $88.2 \%$ & $80.0 \%$ \\
\hline & No & 2 & 15 & & & & & \\
\hline
\end{tabular}

PPV: positive predict value; NPV: negative predict value

variables were used to investigate predictive combinations for adrenal metastasis. The highest accuracy for predicting adrenal metastasis was for the combination of "SUV ${ }_{\text {max }}$ ratio $>1.25$ or metastasis in other positions" with a $79.2 \%, 91.4 \%$ and $59.2 \%$ of accuracy, sensitivity and specificity, respectively (Table 5). The combinations of the variables $\mathrm{SUV}_{\max }$ ratio $>0$ or metastasis in other positions or age $<60$ " had the highest sensitivity of $98.8 \%$, but a relative lower accuracy of $70.8 \%$ and specificity of $24.5 \%$.
In multivariate analysis of patients with lung cancer, the $\mathrm{SUV}_{\max }$ ratio and metastasis in other positions were the two independent impact factors for adrenal metastasis. The criteria of an $\mathrm{SUV}_{\max }$ ratio $>0$ or metastasis in other positions had the highest sensitivity of $96.6 \%$, lowest specificity of $46.4 \%$, and highest accuracy of $84.3 \%$. For patients with gastrointestinal cancer, the criteria of an $\mathrm{SUV}_{\max }$ ratio $>1.25$ or age $<55$ had the highest sensitivity of $86.7 \%$, the second highest specificity of $85.0 \%$, and the highest accuracy of $85.7 \%$ (Table 5). 


\section{Discussion}

This retrospective study used FDG PET/CT to evaluate characteristics of adrenal lesions in patients with non-adrenal primary tumor and with unknown cancer. It found that subjects with a known primary tumor were more likely to have an adrenal tumor mass than subjects with unknown primary tumor. However, both cohorts of subjects had similar frequency of other abnormalities including nodules and enlarged adrenals, suggesting these characteristics are not good predictors of the presence of adrenal lesion or adrenal cancer.

Prior studies have found that the use of the SUV ${ }_{\text {max }}$ ratio is highly accurate in distinguishing between benign and malignant adrenal tumors $[4,8-13]$. These studies found that the sensitivity, specificity, and accuracy for distinguishing benign from cancerous tumors were between $85 \%$ to $100 \%$. Similarly, this study found that FDG PET/CT was efficient at distinguishing between benign and malignant adrenal cancers. It found that about $40 \%$ of patients with primary tumors did not have a malignant adrenal lesion which is similar to what has been previously described [1]. Moreover, the adrenal lesions in patients without cancer were all benign or due to adrenal hyperplasia which is consistent with the low frequency (about $10 \%$ ) of primary adrenal tumors [1]. Only $1.9 \%$ and $1.1 \%$ of the subjects with no primary tumor or with a primary tumor but no adrenal metastasis, respectively, had a SUV ${ }_{\text {max }}$ ratio > 2.5. In patients with a primary tumor, the left adrenal had a higher frequency of a metastatic tumor mass than the left (50.6\% versus $33.3 \%$ ). The reasons for this are unclear.

The most common malignant lesions that metastasize to the adrenal gland include malignant melanomas, kidney, liver, gastroinitestinal, breast, colon, lung, and bronchial carcinomas [1]. In this study, the most common primary cancer that metastasized to the adrenal gland was lung cancer (53.7\%). The majority of subjects with adrenal metastasis also had metastatic cancer in other body organs (75.3\%) and this was even higher for the sub-group of patients with gastrointestinal primary cancer (93.3\%). These findings are consistent with patients in this study who had adrenal metastasis having advanced cancer.

Univariate analysis found that age, the $\mathrm{SUV}_{\max }$ ratio and the presence of metastasized cancer in other organs were independent predictors for adrenal metastasis in patients with a non-adrenal primary tumor. The risk of metastasized adrenal cancer from a primary tumor decreased with every increasing year of age. These same variables were independent predictors of adrenal metastasis in patients with primary tumors in the lung and gastrointestinal track. Multivariate analysis found that for patients with primary tumors that having a $\mathrm{SUV}_{\max }$ ratio $>1.25$ and the presence of metastasis in other organs had a high sensitivity, specificity, and accuracy for predicting adrenal metastasis. For patients with lung cancer the variables of the $\mathrm{SUV}_{\max }$ ratio $>0$ and multiple metastasis sited and for patients with gastrointestinal cancer the variables of a $\mathrm{SUV}_{\mathrm{m}}$ ratio $>1.25$ and age of $<55$ years were the best combination of sensitivity, specificity and accuracy in predicting the presence of adrenal metastasis.

The major limitation of this study is that it was a retrospective study. Also, our FDG PET/CT analysis was solely quantitative and several studies have found that qualitative analysis of FDG PET/CT may be more accurate in distinguishing between malignant and benign adrenal lesions $[10,12]$. The size of the study was small $(\mathrm{N}=371)$ and subjects with a primary lesion in the lung accounted for almost $50 \%$ of this population. Hence, the findings of the total population with primary tumors may predominately reflect the characteristics of the lung cancer population. Larger studies are necessary that include sizeable populations that represent multiple primary tumor sites are needed to more fully assess the parameters that predict adrenal metastasis.

In summary, FDG PET/CT was useful in characterizing adrenal lesions present in patients with and without known primary cancers. It identified variables that may useful in assessing the risk of developing adrenal metastasis. The use of FDG PET/CT may help facilitate the physician in diagnosis and determining the optimum treatment strategies in cancer patients.

Acknowledgment: We are in debt to Drs. Rui-Min Wang, ChangBin Liu, Da-Yi Yin, Shu-Lin Yao of Department of Nuclear Medicine, Chinese PLA General Hospital for critical reading. This study was not financially supported by any institution and commercial sources.

\section{References}

[1] MCLEAN K, LILIENFELD H, CARACCIOLO JT, HOFFE S, TOURTELOT JB et al. Management of isolated adrenal lesions in cancer patients. Cancer Control 2011; 2: 113-26.

[2] YOUNG WF The incidentally discovered adrenal mass. NEJM 2007; 356:601-9. http://dx.doi.org/10.1056/NEJMcp065470

[3] UBEROI J, MUNVER R Surgical management of metastasis to the adrenal gland: open laparoscopic, and ablative approaches. Curr Urol Rep 2009; 10: 67-72. http://dx.doi.org/10.1007/ s11934-009-0012-0

[4] LU Y, XIE D, HUAUNG W, GONG H, YU J 18F-FDG PET/CT in the evaluation of adrenal masses in lung cancer patients. Neoplasm 2010; 57: 129-34. http://dx.doi.org/10.4149/neo $2010 \quad 02 \_129$

[5] CAOILI EM, KOROBKIN M, FRANCIS IR, COHAN RH, PLATT JF et al. Adrenal masses: characterization with combined unenhanced and delayed enhanced CT. Radiology 2002; 222: 629-33. http://dx.doi.org/10.1148/radiol.2223010766

[6] BAE KT, FUANGTHARNTHIP P, PRASAD SR, JOE BN, HEIKEN JP Adrenal masses: CT characterization with histogram analysis method. Radiology 2003; 228: 735-42. http://dx.doi.org/10.1148/radiol.2283020878

[7] TSUSHIMA Y, ISHIZAKA H, MATSUMOTO M Adrenal masses: differentiation with chemical shift fast low-angle shot MR imaging. Radiology 1993; 186: 705-9

[8] OKADA M, SHIMONO T, KOMEYA Y, ANDO R, KAGAWA $\mathrm{Y}$ et al. Adrenal masses; the value of additional fluorodeoxyglucose-positron emission tomography/computed tomography 
(FDG-PET/CT) in differentiating between benign and malignant lesion. Ann Nucl Med 2009; 23: 349-54. http://dx.doi. org/10.1007/s12149-009-0246-4

[9] GRATZ S, KEMKE B, KAISER W, HEINIS J, BEHR TM et al. Incidental non-secreting adrenal masses in cancer patients: intra-individual comparison of $18 \mathrm{~F}$-fluorodeoxyglucose positron emission tomography/computed tomography with computed tomography and shift magnetic resonance imaging. J Int Med Res 2010; 38: 633-44.

[10] TESSONNIER L, SEBAG F, PALAZZO FF, COLAVOLPE C, DE MICCO C et al. Does 18F-FDG PET/CT add diagnostic accuracy in incidentally identified non-secreting adrenal tumours ? Eur J Ncul Med Mol Imaging. 2008; 35: 2018-25. http://dx.doi.org/10.1007/s00259-008-0849-3
[11] ANSQUER C, SCIGLIANO S, MIRALLIE E, TAIEB D, BRUNAUD L et al. 18F-FDG PET/CT in the characterization and surgical decision concerning adrenal masses: a prospective multicentre evaluation. Eur J Nucl Med Mol Imaging 2010; 37: 1669-78. http://dx.doi.org/10.1007/s00259-010-1471-8

[12] PARK BK, KIM CK, KIM B, CHOI JY. Comparison of delayed enhanced CT and 18F-FDG PET/CT in the evaluation of adrenal masses in oncology patients. J Comput Assist Tomogr 2007; 31: 550-6. http://dx.doi.org/10.1097/rct.0b013e31802fa8e1

[13] BOLAND GWL, BLAKE MA, HOLALKERE NS, HAHN PF. $\mathrm{PET} / \mathrm{CT}$ for the characterization of adrenal masses in patients with cancer: qualitative versus quantitative accuracy in 150 consecutive patients. AJR 2009; 192: 956-62. http://dx.doi. org/10.2214/AJR.08.1431 